\title{
The MicroRNAs and Stroke: No Need to be Coded to be Counted
}

\author{
Raghu Vemuganti
}

Published online: 28 May 2010

(C) Springer Science+Business Media, LLC 2010

Experimental stroke research conducted in the past three decades can be divided into two major categories. The first is the molecular and cellular studies that evaluated the mechanisms of stroke-induced neuronal death and the second is the development and testing of putative therapeutic compounds to prevent post-stroke brain damage and neurologic dysfunction. Both categories of studies focused on evaluating and/or targeting specific proteins or the genes that encode those proteins. However, protein-coding genes represent $<2 \%$ of the eukaryotic genome as $\sim 98 \%$ of the transcriptional output is non-coding (nc) RNAs that include microRNA (miRNA), small interfering RNA (siRNA), piwi-interacting RNA (piRNA), long non-coding RNA (LncRNA), small nuclear RNA (snRNA), small nucleolar RNA (snoRNA), antisense RNA (asRNA), and Y-RNA [1]. The ncRNAs are currently considered as the master controllers of the transcription and translation that decides the organ- and cell-specific protein repertoire [2]. Hence, any disruption in the ncRNA function could lead to severe compromises in cellular homeostasis. Despite their abundance and paramount functional importance, very few studies to date evaluated the significance of ncRNAs in acute brain damage.

The miRNAs (18 to 24 nucleotides long) are the most studied of all classes of ncRNAs. The miRNAs are known to be transcribed from specific genes located in the introns, exons, as well as the intergenic regions of the genome. To date, the number of miRNAs identified in different organisms is minimal. For example, only $940 \mathrm{miRNAs}$ were identified in humans and 326 miRNAs were identified in rats (www.

R. Vemuganti $(\square)$

Department of Neurological Surgery, University of Wisconsin, Madison, WI 53792, USA

e-mail: vemugant@neurosurgery.wisc.edu
miRBase.com). The miRNAs are evolutionarily well conserved and binds to specific 8-nt complimentary seed sequences in the $3^{\prime} \mathrm{UTR}$ s of mRNAs. Binding of a miRNA induces either mRNA degradation or translational arrest [3]. As a miRNA can bind to multiple mRNAs and most mRNAs contain seed sequences for multiple miRNAs, the handful of miRNAs can effectively control the multitude of mRNAs in the body.

The miRNA dysfunction is suspected to be a contributing factor for many CNS pathologies including brain tumors [4], Alzheimer's disease [5], Down's syndrome [6], schizophrenia [7], and stroke [8]. Recent studies showed that focal or global ischemia in adult rodents leads to rapid and sustained changes in the cerebral miRNA profiles [9-11]. It is well known that $3 \%$ to $4 \%$ of the cerebral mRNAs alter during the acute phase ( $2 \mathrm{~h}$ to 3 days) following focal ischemia [12-15], but strikingly $>20 \%$ of the miRNAs alter in the ischemic brain, indicating the possibility that ncRNA genes are more susceptible to stroke than protein-coding genes. As the transcription of mRNA and miRNA genes might be independently influenced by stroke, a higher magnitude of change in miRNAs might serve as an extra layer of control to prevent aberrant protein expression under disease conditions.

Many individual stroke-responsive miRNAs were shown to play a role in mediating the secondary brain damage and functional outcome after experimental stroke. It was shown that mir-145 will be upregulated to eightfold in rat brain following focal ischemia [10]. A major target of the mir145 is the antioxidant enzyme superoxide dismutase-2 (SOD-2) mRNA and presumably mir-145 prevents SOD-2 protein translation after stroke. When the ischemic rats were treated with antagomir-145, there was an increased expression of SOD-2 in the cortical neurons and a significantly decreased infarction presumably by curtailing the oxidative stress [10]. Another recent study showed that induction of 
mir-497 following focal ischemia promotes ischemic neuronal death by negatively regulating antiapoptotic proteins, bcl-2 and bcl-w [16]. Activation of the transcription factor peroxisome proliferator-activated receptor (PPAR)- $\delta$ induces vascular endothelial cell protection and this beneficial effect was shown to be mediated by preventing the post-ischemic induction of miR-15a which targets bcl-2 [17]. The miRNAs are also thought to promote neuroprotection. Importantly, miRNAs that target mRNAs which code for transcription factors like MeCP2 play a critical role in inducing ischemic tolerance in mouse brain [18]. Ischemic preconditioning was also shown to modulate miRNAs which are upstream to neuroprotective signaling pathways in rat brain [19].

It is interesting to note that miRNA profiles alter rapidly in blood following stroke and intracerebral hemorrhage in rodents $[9,20]$. Altered miRNA profiles were also reported in stroke patients [8]. This study showed that many miRNAs that are expressed at a low level in normal human blood were observed to be highly expressed in stroke samples irrespective of etiology. These authors also showed that different stroke subtypes show distinctly different blood miRNA profiles and they could separate the small artery and large artery stroke samples based on miRNA profiles [20]. In addition, the blood miRNAs of the patients showing good outcome and bad outcome after stroke separated into distinct clusters [20]. These studies indicate that blood miRNAs can be used as genomic biomarkers to quickly identify stroke, stroke subtypes, and possibly the outcome after a therapy.

The current view is that miRNAs play a more diverse role than just silencing mRNAs as certain miRNAs can also upregulate translation [21]. The 8-bp seed sequences that miRNAs recognize in the $3^{\prime}$ UTR of mRNAs can be found in several genomic locations. The miRNA mir-379 was shown to bind to the E-cadherin promoter, leading to the induction of E-cadherin gene expression [22]. This phenomenon known as RNA-induced gene activation might also play an important role in post-ischemic gene induction. Using in silico analysis, we observed that eight miRNAs upregulated after focal ischemia can bind to the complementary seed sequences in 877 promoters in rat genome [10]. Furthermore, it was shown that, if the levels of a specific mRNAs are increased, that mRNA can instruct the expression of an upstream miRNA to prevent its own translation as a feedforward mechanism [23]. Recent studies from our laboratory showed that miRNA promoters contain several transcription factor binding sites and treating rats with rosiglitazone (a high-affinity PPAR $\gamma$ agonist) alters the cerebral expression of miRNAs that contain PPAR binding sites [24]. Thus, miRNAs and mRNAs can control each other bi-directionally and hence their relationship is complex.

To conclude, there is much excitement in understanding the functional significance of miRNAs to ischemic brain damage and the studies conducted so far is just the tip of the iceberg. Importantly, an altered miRNA expression can have profound consequences on transcription as well as translation. As new functions of miRNAs as well as other ncRNAs are still emerging, in the future some of those can be identified as good therapeutic targets to prevent brain damage in stroke patients. Financial support was provided by NIH grant NS061071.

\section{References}

1. Taft RJ, Pang KC, Mercer TR, Dinger M, Mattick JS. Non-coding RNAs: regulators of disease. J Pathol. 2010;220:126-39.

2. Jacquier A. The complex eukaryotic transcriptome: unexpected pervasive transcription and novel small RNAs. Nat Rev Genet. 2009; $10: 833-44$.

3. Jackson RJ, Hellen CU, Pestova TV. The mechanism of eukaryotic translation initiation and principles of its regulation. Nat Rev Mol Cell Biol. 2010;11:113-27.

4. Nicoloso MS, Calin GA. MicroRNA involvement in brain tumors: from bench to bedside. Brain Pathol. 2008;18:122-9.

5. Hébert SS, Horré K, Nicolaï L, Papadopoulou AS, Mandemakers W, Silahtaroglu AN, et al. Loss of microRNA cluster miR-29a/b-1 in sporadic Alzheimer's disease correlates with increased BACE1/betasecretase expression. Proc Natl Acad Sci USA. 2008;105: 6415-20.

6. Kuhn DE, Nuovo GJ, Martin MM, Malana GE, Pleister AP, Jiang $\mathrm{J}$, et al. Human chromosome 21-derived miRNAs are overexpressed in Down syndrome brains and hearts. Biochem Biophys Res Commun. 2008;370:473-7.

7. Beveridge NJ, Tooney PA, Carroll AP, Gardiner E, Bowden N, Scott RJ, et al. Dysregulation of miRNA $181 \mathrm{~b}$ in the temporal cortex in schizophrenia. Hum Mol Genet. 2008;17:1156-68.

8. Tan KS, Armugam A, Sepramaniam S, Lim KY, Setyowati KD, Wang $\mathrm{CW}$, et al. Expression profile of microRNAs in young stroke patients. PLoS One. 2009;4:e7689.

9. Jeyaseelan K, Lim KY, Armugam A. MicroRNA expression in the blood and brain of rats subjected to transient focal ischemia by middle cerebral artery occlusion. Stroke. 2008;39:959-66.

10. Dharap A, Bowen K, Place R, Li LC, Vemuganti R. Transient focal ischemia induces extensive temporal changes in rat cerebral microRNAome. J Cereb Blood Flow Metab. 2009;29:675-87.

11. Yuan Y, Wang JY, Xu LY, Cai R, Chen Z, Luo BY. MicroRNA expression changes in the hippocampi of rats subjected to global ischemia. J Clin Neurosci. 2010;17:774-8.

12. Soriano MA, Tessier M, Certa U, Gill R. Parallel gene expression monitoring using oligonucleotide probe arrays of multiple transcripts with an animal model of focal ischemia. J Cereb Blood Flow Metab. 2000;20:1045-55.

13. Vemuganti R, Bowen KK, Dhodda VK, Song G, Franklin JL, Gavva NR, et al. Gene expression analysis of spontaneously hypertensive rat cerebral cortex following transient focal cerebral ischemia. J Neurochem. 2002;83:1072-86.

14. Lu A, Tang Y, Ran R, Clark JF, Aronow BJ, Sharp FR. Genomics of the periinfarction cortex after focal cerebral ischemia. J Cereb Blood Flow Metab. 2003;23:786-810.

15. Sharp FR, Lit L, Xu H, Apperson M, Walker W, Wong B, et al. Genomics of brain and blood: progress and pitfalls. Epilepsia. 2006;47:1603-7.

16. Yin KJ, Deng Z, Huang H, Hamblin M, Xie C, Zhang J, et al. miR-497 regulates neuronal death in mouse brain after transient focal cerebral ischemia. Neurobiol Dis. 2010;38:17-26.

17. Yin KJ, Deng Z, Hamblin M, Xiang Y, Huang H, Zhang J, et al. Peroxisome proliferator-activated receptor delta regulation of 
miR-15a in ischemia-induced cerebral vascular endothelial injury. J Neurosci. 2010;30:6398-408.

18. Lusardi TA, Farr CD, Faulkner CL, Pignataro G, Yang T, Lan J, et al. Ischemic preconditioning regulates expression of microRNAs and a predicted target, $\mathrm{MeCP} 2$, in mouse cortex. J Cereb Blood Flow Metab. 2010;30:744-56.

19. Dharap A, Vemuganti R. Ischemic preconditioning alters cerebral microRNAs that are upstream to neuroprotective signaling pathways. J Neurochem. 2010 (in press).

20. Liu DZ, Tian Y, Ander BP, Xu H, Stamova BS, Zhan X, et al. Brain and blood microRNA expression profiling of ischemic stroke, intracerebral hemorrhage, and kainate seizures. J Cereb Blood Flow Metab. 2010;30:92-101.
21. Vasudevan S, Tong Y, Steitz JA. Switching from repression to activation: microRNAs can up-regulate translation. Science. 2007;318:1931-4.

22. Place RF, Li LC, Pookot D, Noonan EJ, Dahiya R. MicroRNA373 induces expression of genes with complementary promoter sequences. Proc Natl Acad Sci USA. 2008;105:1608-13.

23. Taganov KD, Boldin MP, Chang K-J, Baltimore D. NF-kBdependent induction of microRNA mir-146, an inhibitor targeted to signaling proteins of innate immune response. Proc Natl Acad Sci USA. 2006;103:12481-6.

24. Vemuganti R, Dharap A. Transcription factor PPAR-gamma controls microRNA expression in rat brain. Trans Am Soc Neurochem. 2010;41:86. 